\title{
WORKSHOP E O DESIGN PARTICIPATIVO: UMA PERSPECTIVA DA COLABORAÇÃO DESIGNER-CLIENTE
}

Marcelo C. Halpern - UFRGS | marcelohalpern@gmail.com Filipe C. X. da Costa - UNISINOS | fcampelo@unisinos.br 
O presente trabalho se propõe a discutir o impacto da presença de usuários/clientes em uma prática projetual de workshop. Através de uma pesquisa bibliográfica e um estudo de caso foi estabelecida uma relação entre tópicos como design thinking, codesign e ferramentas metaprojetuais como o workshop. Como resultado, é sugerido que a atuação de clientes de um contexto de projeto pode influenciar positivamente os resultados de uma prática projetual. Portanto, propõe-se que em determinados contextos as empresas e clientes possam vir a se aproximar do processo de projetação com o objetivo de potencializar a capacidade de sua implementação.

Palavras-chave: Workshop, Metaprojeto, CoDe$\boldsymbol{\alpha}$ sign, Design Thinking.

This present paper proposes to discuss the impact of the presence of users / clients whilist in the workshop design practice. Through a bibliographical research and a case study, this work establishes a relation between topics as design thinking, codesign and metaprojectual tools like the workshop practice. As a result, it is suggested that client engagement in a project context can positively influence the results of design practice. Therefore, it is proposed that in certain contexts, companies and clients may come closer to the design process in order to enhance their capacity towards implementation.

Keywords: Workshop, Metaproject, CoDesign, Design Thinking. 
1.

INTRODUÇÃO
Historicamente as atividades e competências dos designers são essencialmente relacionadas à solução de problemas em níveis de projetação industrial. No entanto, ao introduzir o conceito de Design Estratégico, Zurlo (2010) passa a discutir uma reavaliação das responsabilidades dos designers diante de uma ótica estratégica e gerencial. Este fato expõe uma aproximação inevitável da prática projetual com empresas e organizações com foco na interpretação da complexidade e desenvolvimento de projetos orientados em rumos e cenários estratégicos. Ao se posicionar em um patamar estratégico junto ao processo de tomada de decisões, a avaliação e estudo das atividades envolvendo a prática de design também passam a ser investigadas com mais profundidade.

Um dos aspectos mais emblemáticos da discussão a respeito da aproximação do design com organizações é a influência de usuários em relação a abordagens projetivas. Conceitos como o do design thinking endereçam características importantes sobre a forma de operação e projetação de designers diante de cenários problemáticos utilizando-se do conhecimento e investigação de contextos práticos, sociais e culturais dos usuários. No entanto, de acordo com Sanders e
Stappers (2008) nos últimos quarenta anos vem sendo discutida a transição de modelos que enquadram usuários como elementos externos da prática projetual, para processos que os posicionam como coautores. Dentre as inúmeras técnicas que permitem o exercício da co-projetação está o workshop. O workshop é descrito por Scaletsky (2008) como um recurso metaprojetual projetual coletivo para o desenvolvimento e investigação de cenários-problema.

A partir dessa perspectiva, o objetivo desse artigo é realizar uma discussão a respeito da presença de usuários clientes em uma prática projetual de workshop. Para tanto, o artigo é constituído por uma revisão bibliográfica visando aprofundamento teórico sobre as temáticas de design thinking, design participativo e o workshop como técnica metaprojetual. Além disso, será utilizado um estudo de caso de um workshop com o intuito de se obter insumos práticos e percepções envolvendo a atividade. $\mathrm{O}$ estudo de caso é resultado de um exercício realizado pela turma de mestrado do programa de pós-graduação em Design da Unisinos, e incorporou a temática da educação através da orientação da iniciativa de design social do Dream:In. A principal hipótese levantada neste trabalho é que, em determinados contextos, a presença e participação ativa de clientes em cenários de projetação pode trazer benefícios práticos no desenvolvimento de soluções. Como resultado desta reflexão, espera-se apresentar os principais aspectos e impacto envolvendo a presença de usuários clientes em um formato colaborativo no desenvolvimento de soluções na atividade. 


\section{2.}

PENSANDO

DESIGNE PARTICIPAÇÃO
São muitas as correntes de pensamento que analisam a forma e abordagens de designers em relação ao enfrentamento projetual. O termo design thinking é definido por Cross (2011) como a forma com que designers processam e agem criativamente na solução de problemas. De acordo com Brown (2008), pode ser descrito como um conjunto de ferramentas, métodos e técnicas baseadas no design e incorporadas à cultura de organizações, comunidades e sistemas visando à solução de problemas através de inovação. A efetividade destes processos está diretamente relacionada a visões orientadas pela observação e interpretação de contextos sociais, técnicos e culturais de comunidades e indivíduos. Por causa destas características, a abordagem do design thinking vem sendo amplamente difundida em diversos cenários, dentre eles no âmbito de projetos sociais. Segundo Brown (2008), a abordagem é também reconhecida por ser um processo não-linear de caráter exploratório, tendo suas principais estruturas de organização formadas por três fases: inspiração, ideação e implementação. A primeira etapa, Inspiração, diz respeito a abordagem, motivação, identificação de cenários e compreensão de problemas. Em seguida, a fase de Ideação envolve diretamente o desenvolvimento do trabalho, sendo composta por geração de alternativas, prototipagem e testagem e desenvolvimento do projeto. Por fim, a Implementação diz respeito à viabilização, à execução e ao encaminhamento dos projetos ao mercado. Outra característica essencial do design thinking é a sua abordagem multidisciplinar. De acordo com Brown (2008), a resolução de problemas com base no design thinking envolve a interação entre membros de equipes heterogêneas, promovendo uma maior troca de experiências assim como a contribuição de diferentes perspectivas culturais e técnicas na concepção e gestão efetiva de projetos.

No entanto, de acordo com Sanders e Stappers (2008), a evolução de modelos de pesquisa e prática em design centrados estritamente na observação e avaliação de contextos de usuário vêm sendo significativamente repensados para perfis mais participativos. Além da remodelagem dos papéis envolvendo as competências dos designers, são muitos os esforços a fim de impulsionar a uma realocação fundamental das atividades dos usuários tornando-os ativos em um contexto projetual como co-criadores. Os primeiros registros de motivações e publicações envolvendo o codesign tem sua origem no norte da Europa, mais especificamente na Escandinávia na década de 70. Foi na conferência da Design Research Society de 1971 que foram iniciadas as primeiras discussões formais sobre a necessidade de uma revisão nos modelos unilaterais de projetação com usuários. Nos procedimentos do encontro, Nigel Cross (1971) e Sanders e Stappers 
(2008) acusaram a obsolescência dos processos vigentes, e definiram como inevitável e providencial para a saúde da área de design uma transição aguda para modelos inclusivos entre designers e usuários em regime de participação e coautoria. A partir desta perspectiva, de acordo com Binder, Brandt e Gregory (2008), cada vez mais o envolvimento de usuários ativamente presentes no processo de desenvolvimento de projetos tem se tornado uma realidade dentro de organizações e empresas.
3.

DESIGN

ESTRATÉGICO

E WORKSHOP
Diante das significativas mudanças em cenários de atuação do designer, Zurlo (2010) e Franzato (2010) evidenciam uma importante transição nos modelos tradicionais de relacionamento entre as organizações e o design. Esta transição é reflexo de novas abordagens e exigências das organizações sobre as competências do design, sendo substituídos perfis antes orientados à projetação industrial por uma dimensão de atuação estratégica. Diante disso, Mozotta (2011) defende que dentre as principais atribuições e competências contemporâneas do design encontram-se características de atuação em altos patamares gerenciais em canais econômicos, criativos, sistemáticos, de planejamento e idealização de diferenciais competitivos. Nessa perspectiva, Franzato (2010) apresenta que o design estratégico agrupa as principais características em termos de evolução das abordagens do design diante do relacionamento com empresas e organizações. A partir disso, encaminha-se para a conceituação de Design Estratégico como uma abordagem do design a um determinado sistema-produto, baseado em atividades de projetação orientadas por um regime de inovação (design-driven), estruturadas sobre um processo com base metaprojetual e conduzidas por um grupo de competências específicas, visando resultados estratégicos, como definem Francesco Zurlo (2010) e Anna Meroni (2008). 
Diante da perspectiva do design estratégico, o workshop é amplamente discutido como um recurso projetual voltado ao desenvolvimento de conceitos de projeto e orientados por um briefing. As atividades de workshop têm caráter essencialmente coletivo e multidisciplinar, características defendidas pela lógica do codesign e design thinking, já que envolvem a colaboração de indivíduos com diferentes bagagens culturais e competências distintas. De acordo com Scaletsky (2008), um workshop pode ser classificado como uma ferramenta coletiva e multifacetada de caráter criativo que visa a geração de ideias e alternativas diante de um cenário problema. Em sua definição apresenta o workshop como "um momento de imersão criativa, de lançamento de ideias que busca, através de técnicas variadas, conduzir a formulação de cenários de projeto, criação de conceitos ou mesmo a proposição das primeiras ideias concretas que respondam ao briefing" (SCALETKSY, 2008, p. 1135). Mesmo com diversas perspectivas, Fraga (2011) afirma que a técnica de workshop é amplamente utilizada por diferentes áreas, tanto em níveis acadêmicos quanto profissionais, como um subsídio estratégico com o intuito de promover a reflexão coletiva em torno de um mesmo contexto, problema ou investigação. A característica multifacetada dos workshops pode ser descrita pela sua flexibilidade em compor técnicas e métodos em seu exercício. Recursos metaprojetuais, partes integrantes do portfólio de técnicas do design estratégico, como brainstorms, moodboards, construção de cenários, pesquisa blue sky, storyboards entre outros, são amplamente aplicados em workshops.

\section{4.}

DESIGN

ESTRATÉGICO

E WORKSHOP
Dentre os diversos projetos sociais que utilizam o design thinking como base estrutural, pode-se citar o Dream:In. A iniciativa fundada em 2011 na Índia por um grupo de instituições, empresas e organizações não governamentais, tinha por objetivo: "identificação, desenvolvimento e implementação de políticas públicas e negócios empreendedores, com a aplicação de um método inovador e de dinâmica distinta" (DREAMIN, 2012). O êxito e interesse pelo programa fez com que surgissem diversas iniciativas derivadas e semelhantes ao redor do mundo. O Dream:In é conduzido por temáticas e é essencialmente estruturado por três bases de ação fundamentais: sonhar, acreditar e realizar (Figura 1). A primeira etapa, sonhar, é composta pela captura audiovisual de "sonhos", depoimentos representados por desejos, anseios e aspirações, através da realização de entrevistas em profundidade com indivíduos. Na fase "acreditar", estes sonhos são compilados, triados e agrupados por especialistas e curadores técnicos com base em suas características, e manipulados visando sua viabilidade e oportunidades de execução. A etapa "realizar" 
envolve a aproximação dos "sonhadores" com investidores e organizações visando a avaliação das oportunidades geradas e sua adoção e implementação prática (DREAMIN, 2012).

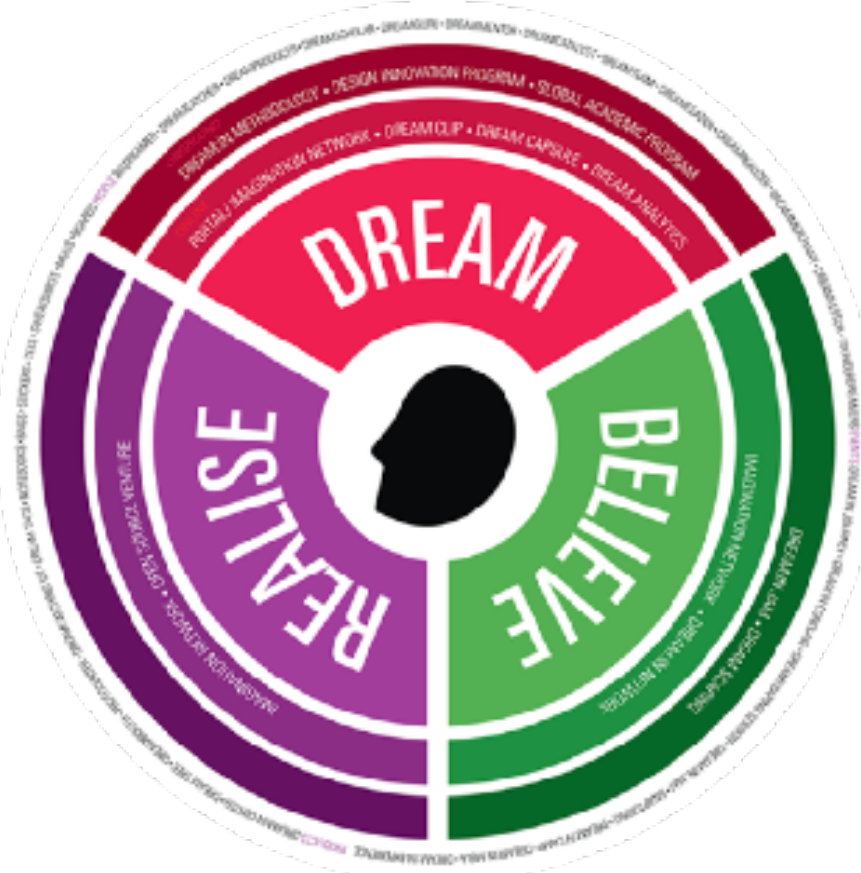

Figura 1: Bases de ação fundamentais Fonte: DREAM:IN (2016).

A fim de ilustrar a lógica do workshop e as características de um processo colaborativo de codesign, buscou-se, através da exposição de um estudo de caso real, apresentar as principais conclusões relacionadas com a importância de usuários e clientes como participantes no desenvolvimento de projetos. O caso descrito é resultado da disciplina de Laboratório de Experimentos em Design do programa pós-graduação em Design da Universidade do Vale do Rio dos Sinos. O workshop foi desenvolvido por quatro grupos de alunos da turma de Mestrado, e tinha por objetivo o desenvolvimento de projetos sobre a temática da educação orientado pela lógica do projeto Dream:In. Cada um dos grupos formados por designers, coordenadores e especialistas desenvolveram dinâmicas integrantes do projeto Dream:In, no entanto, os grupos receberam autonomia por parte dos docentes coordenadores para avaliar características distintas do processo (Figura 2). Portanto, observa-se que o objetivo deste trabalho não é realizar-se uma avaliação crítica a respeito dos processos e estruturas específicas do projeto Dream:In, mas utilizar-se da prática em que foi envolvido a fim de se construir uma discussão a respeito da colaboração em workshops.

Figura 2: Estrutura dos grupos. Fonte: elaborado pelo autor.
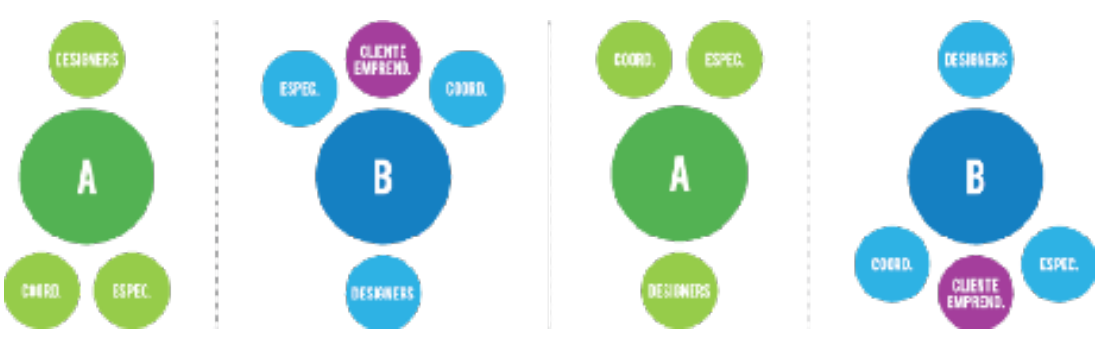

Diante das várias abordagens de investigação possíveis em um workshop, foi escolhida a realização de uma análise a respeito da colaboração envolvendo não só usuários, mas a participação específica de indivíduos caracterizados como 
clientes em um cenário colaborativo de projeto. Esta definição de objeto de estudo orientou o perfil de observação durante a realização de todo o workshop e contou com a condução de entrevistas com membros de todas as equipes assim como a análise dos registros e captações audiovisuais dos grupos. Outro fator decisivo que permitiu a realização do estudo foi a estrutura das equipes. Apesar de serem multidisciplinares, apenas dois dos quatro grupos contaram com a presença de clientes como membros de suas esquipes, fato que permitiu a realização de um comparativo entre as dinâmicas e resultados. A escolha pela definição do conceito de "cliente" neste contexto deu-se através da lógica do projeto Dream:In, entendendo-se que os clientes finais da fase de geração de ideias são caracterizados por empreendedores. Observa-se que mesmo tendo como foco de estudo definido, os membros clientes não foram instruídos a adotar posturas específicas ou predeterminadas, isso se deu pelo fato de que análises desejadas de suas contribuições deveriam ser feitas com o máximo de naturalidade possível.
4.

APRESENTAÇÃO

DOS RESULTADOS
A dinâmica do workshop foi conduzida através de pequenas sessões de insight, onde os grupos eram estimulados através de clipes audiovisuais (Figura 3) no formato estabelecido pelo Dream:In e depois recebiam um determinado tempo para a realização de brainstorms e debates a fim de se chegar a ideias e soluções para projetos envolvendo o tema sugerido. Ao se observar a prática da atividade realizada foi possível evidenciar o impacto da presença (ou ausência) dos clientes na estrutura das equipes em diferentes níveis. Um dos níveis a ser analisado diz respeito ao foco dos insights e ideias. Enquanto os grupos sem integrantes clientes (A) apresentaram uma postura mais orgânica, abstrata e conceitual na forma de conduzir as ideias, as equipes com integrantes clientes (B) se mostraram mais pragmáticas e objetivas na definição de suas soluções, com metas e objetivos. Foi possível identificar que a presença de empreendedores orientou os grupos a se enquadrarem em um caráter mais prático, 
5.
Mesmo com um objeto de estudo bastante pontual, foi possível, com base em observações da prática do workshop, realizar um levantamento sobre uma série de questões relacionados à estrutura da atividade quando envolvendo clientes finais como participantes. Por serem estruturados por características multidisciplinares e coletivas, os workshops são ferramentas que dependem de diferentes agentes para o sucesso no processo de construção de projetos. No entanto, é sabido que assim como para qualquer técnica projetual, não existem fórmulas absolutas ou lineares para o desenvolvimento de atividades como o workshop, portanto, os casos devem ser analisados com olhares específicos. A condução da prática deve estar relacionada e ser condizente ao tema abordado assim como ao interesse de estudo. No estudo de caso do workshop, a presença de um agente empreendedor foi utilizada como um foco de estudo específico. Isso se deu pelo fato de que neste contexto, estes agentes ocuparam o papel de clientes/consumidores, seguindo a lógica do projeto Dream:In. Como descrito anteriormente, os resultados das pesquisas realizadas apontaram para pontos importantes da atuação dos clientes neste contexto. Ao ocuparem uma posição de críticos, delineadores e balizadores dos projetos, ficou evidente que o papel destes indivíduos pode ter influenciado a forma com que as soluções foram estruturadas. Com base nisso, como ponto positivo, pode-se sugerir que esta influência motivou uma aproximação de conceitos de projeto a uma realidade mercadológica mais próxima da implementação. Por outro lado, a presença destes indivíduos, em uma perspectiva de geração de alternativas, pode ter sido prejudicial para o rompimento de características projetuais usuais, e consequentemente um comprometimento com diretrizes inovadoras. 
6.

A reavaliação de papéis e competências do Design motivado por transformações das relações entre consumo, empresas e designers tem levado a discussões bastante aprofundadas sobre o impacto que usuários podem ter tanto em resultados como no processo de geração e consolidação de conceitos. A partir das observações realizadas durante o acompanhamento do workshop, foi possível evidenciar uma série de aspectos relacionados com a participação dos clientes. Dentre os tópicos positivos foi uma maior objetivação em relação à definição e estruturação dos problemas. No entanto, foi acusado que a mesma participação pode ter promovido um maior cerceamento em termos de liberdade criativa especialmente no que diz respeito a exercícios não-contextuais. Além disso, o workshop se mostrou uma ferramenta bastante efetiva na avaliação das dinâmicas e processos envolvendo clientes e designers.

Mesmo com um estudo bastante específico e em pequena escala, pode ser sugerido que a prática de colaboração entre designers e clientes pode vir a promover ganhos importantes em determinados cenários de projetação. Diante da lógica estudada é possível constatar que em muitos casos a aproximação de organizações com o processo de projetação acaba ocorrendo em uma etapa demasiadamente tardia. No contexto estudado do projeto Dream:In, acredita-se que os projetos sugeridos se beneficiaram através desta aproximação, já que estreitaram relações entre demandas e interesses, e assim potencializaram a capacidade de implementação. Com base na reflexão desenvolvida, também foi constatada a necessidade de um maior aprofundamento na temática de integração entre clientes e designers em contextos colaborativos. Para tanto se espera que este trabalho possa auxiliar no processo de fomentação de discussões envolvendo o tema do codesign tanto em cenários acadêmicos quanto de mercado. 
REFERÊNCIAS BINDER, T.; BRANDT, E.; GREGORY, J. Design participation(-s). CoDesign, v. 4, n. 1, p. 1-3, 2008.

BROWN, T. Design thinking. Harvard business review, n. June, 2008.

CROSS, N. Design Thinking: Understanding how Designers think and work. New York: Berg, 2011.

CROSS, N. (Ed.). In: Design participation: Proceedings of the design research society's conference 1971, Academy editions, London, UK, 1972.

DREAM:IN. Dream:In Brasil. Disponível em: <http:// www.dreamin.com.br/>. Acesso em: 8/10/2016.

FRAGA, Eliara. Workshop em design: espaços de aprendizagem e geração de conhecimento. Dissertação - Universidade do Vale do Rio dos Sinos. Porto Alegre, 2011.

FRANZATO, C. O design estratégico no diálogo entre cultura de projeto e cultura de empresa. Strategic Design Research Journal, v. 3, n. 3, p. 89-96, 31 dez 2010.
MERONI, A. Strategic design: where are we now? Reflection around the foundations of a recent discipline. Strategic Design Research Journal - Unisinos, v. 1, n. 1, p. 31-38, 2008.

MOZOTA, B. B. Gestão do Design: Usando o design para construir valor de marca e inovação corporativa. Porto Alegre: Bookman, 2011.

SANDERS, E. B.-N.; STAPPERS, P. J. Co-creation and the new landscapes of design. CoDesign, v. 4, n. 1, p. 5-18, 2008.

SCALETSKY, Celso C. Pesquisa aplicada / pesquisa acadêmica - o caso Sander. In: Anais do Oitavo Congresso Brasileiro de Pesquisa \& Desenvolvimento em Design. São Paulo: Centro Universitário SENAC, 2008, p. 1132- 1145.

ZURLO, F. Design Strategico, in AA. VV., Gli spazi e le arti, Volume IV, Opera XXI Secolo, Editore Enciclopedia Treccani, Roma, 2010. 